\title{
Médiévales
}

Langues, Textes, Histoire

\section{La maison de Bourbon, « escolle de vertu et de perfection ». Anne de France, Suzanne de Bourbon et Pierre Martin}

The House of Bourbon, "school of virtue and perfection ». Anne of France, Suzanne of Bourbon and Pierre Martin

\section{Élodie Lequain}

\section{OpenEdition \\ Journals}

Édition électronique

URL : https://journals.openedition.org/medievales/893

DOI : 10.4000/medievales.893

ISSN : $1777-5892$

\section{Éditeur}

Presses universitaires de Vincennes

Édition imprimée

Date de publication : 1 juin 2005

Pagination : $39-54$

ISBN : 2-84292-169-0

ISSN : 0751-2708

\section{Référence électronique}

Élodie Lequain, «La maison de Bourbon, « escolle de vertu et de perfection». Anne de France, Suzanne de Bourbon et Pierre Martin », Médiévales [En ligne], 48 I printemps 2005, mis en ligne le 02 mars 2007, consulté le 24 avril 2022. URL : http://journals.openedition.org/medievales/893 ; DOI : https://doi.org/10.4000/medievales.893

Ce document a été généré automatiquement le 24 avril 2022.

Tous droits réservés 


\title{
La maison de Bourbon, « escolle de vertu et de perfection ». Anne de France, Suzanne de Bourbon et Pierre Martin
}

\author{
The House of Bourbon, "school of virtue and perfection ». Anne of France, \\ Suzanne of Bourbon and Pierre Martin
}

Élodie Lequain

$1 \mathrm{Au}$ Moyen Âge, nombreux sont les textes adressés à la haute noblesse pour lui conseiller un bon comportement. Construits sur l'idée que le prince ne peut pas prétendre gouverner ses sujets s'il ne se gouverne pas correctement lui-même, le genre des miroirs des princes s'enrichit depuis l'époque carolingienne, renouvelant le "processus de codification éthique de la fonction gouvernante $»^{1}$. Ce sont souvent des religieux qui guident les grands dans leur vie d'ici-bas. Le frère prêcheur Vincent de Beauvais adresse son De eruditione filiorum nobilium à la reine Marguerite de Provence vers $1247^{2}$. Gilles de Rome, ermite de Saint-Augustin, compose vers 1279 le fameux De regimine principum pour l'édification du futur Philippe le Bel ${ }^{3}$.

2 L'éducation de la noblesse est parfois assurée par les parents. Des pères prennent la plume comme Geoffroy de la Tour Landry ${ }^{4}$ qui fut précédé dans cette tâche par saint Louis, auteur d'enseignements pour Isabelle et Philippe ${ }^{5}$. Quelques mères écrivent pour leurs enfants ou pour les demoiselles de leur maison. Au Ix $\mathrm{x}^{\mathrm{e}}$ siècle, Dhuoda rédige un Liber manualis pour son fils Guillaume ${ }^{6}$. Dans un manuscrit du XIII ${ }^{e}$ siècle, nous disposons d'un petit traité intitulé Sachiez certainement écrit vraisemblablement par une mère pour sa fille de haute naissance ${ }^{7}$. Au XIV ${ }^{e}$ siècle, la reine de Hongrie Élisabeth de Bosnie serait l'auteur d'un Manuel d'éducation pour ses filles dont nous avons perdu la trace ${ }^{8}$. De même, hormis le témoignage de Jean Bouchet, nous ne connaissons pas l'Instruction des jeunes filles composée par Gabrielle de Bourbon au Xvi ${ }^{\mathrm{e}}$ siècle? 
Parmi ces nombreux témoignages de l'attention que l'on porte au Moyen Âge à la bonne conduite des nobles, le cas de la maison de Bourbon au début du $\mathrm{xvI}^{\mathrm{e}}$ siècle présente l'intérêt de réunir autour d'une même personne, Suzanne de Bourbon, plusieurs textes didactiques. Descendants de saint Louis, proches de la famille royale, forts de possessions nombreuses, les Bourbon ont tout intérêt à faire de leurs héritiers des gouvernants compétents et des épouses vertueuses autant que capables. Suzanne est la fille unique du duc de Bourbon Pierre II et d'Anne de France ${ }^{10}$. À la mort de son père en 1503 , la princesse n'a que douze ans et elle est l'héritière d'une puissante principauté. Dans ces circonstances, la conduite de Suzanne devenait affaire d'État. La princesse doit être une épouse parfaite, une duchesse exemplaire et une mère attentive à l'éducation d'une progéniture que l'on espère masculine. Vers 1505, Anne de France écrit des Enseignements pour sa fille et en 1517, le frère prêcheur Pierre Martin s'attèle à un Traité de l'érudition et de l'enseignement des enfants des nobles qu'il offre à Suzanne. L'un et l'autre cherchent à lui transmettre des conseils adaptés à sa condition de princesse ${ }^{11}$. Suzanne de Bourbon, élève et enseignante

4 Peu de temps avant son mariage avec Charles de Montpensier en mai 1505, Suzanne reçoit les Enseignements composés par sa mère. Veuve depuis 1503, la dame de Beaujeu veille sur les destinées bourbonnaises auprès de sa fille et de son gendre. Afin de préparer au mieux Suzanne au destin d'épouse, Anne rassemble des conseils dictés par une longue expérience et une grande affection pour celle qui est son seul enfant ${ }^{12}$.

En 1517, après douze années d'attente, l'annonce de la grossesse de Suzanne est un soulagement « car vu la difformité et indisposition de la personne de madite dame l'on n'avoit opinion qu'elle portât jamais enfans ${ }^{13}$. Pierre Martin, confesseur d'Anne de France, prépare alors un Traité de l'érudition et de l'enseignement des enfants des nobles ${ }^{14}$. Le texte est conservé à la Bibliothèque Sainte-Geneviève ${ }^{15}$. Le frère recommande son travail à deux personnages. Une lettre en latin est d'abord adressée à Pierre Popillon, chancelier de Bourbon ${ }^{16}$. Après avoir rappelé par des exemples historiques l'importance que revêt l'éducation de la noblesse, le frère lui soumet son traité et lui demande de bien vouloir relire l'ouvrage dont le contenu peut servir à la bonne éducation de la jeunesse du duché ${ }^{17}$. Puis Pierre Martin compose en français le prologue de son traité qu'il dédie à Suzanne de Bourbon en se félicitant de la "joyeuse concepcion et commancement de lignee qui est en vous $»^{18}$. Le traité se divise en douze chapitres et s'achève par une conclusion de nouveau adressée à Suzanne pour l'encourager à « votre lignee bien instruire et conduyre $»^{19}$. Lorsque Pierre Martin entame la rédaction, l'enfant de Suzanne et de Charles n'est problablement pas encore né. La mention de la naissance de François à Moulins, le 4 septembre 1517, figure à la toute fin du manuscrit ${ }^{20}$; elle est suivie d'un psaume en latin appelant la miséricorde divine sur le nouveau-né ${ }^{21}$.

Entre-temps, le manuscrit compile plusieurs autres textes. Faisant suite immédiatement au Traité de l'érudition, on trouve un passage biblique en latin et son commentaire traduit en vulgaire vraisemblablement inspiré des Postilles de Nicolas de Lyre $^{22}$. Le manuscrit se poursuit par une copie des Enseignements de saint Louis à son fils écrits «ce pendent qu'il assiegeoit la ville de Thunice». Il s'agirait de la charte prise dans le trésor du roi Charles V par son secrétaire Gérard de Montaigu ${ }^{23}$. On sait que le texte retrouvé par Montaigu en 1374 fut offert avec un exemplaire des Enseignements à Isabelle au beau-frère de Charles V, le duc Louis II de Bourbon. En 1517 le compilateur pouvait-il encore consulter dans les archives familiales le manuscrit offert au duc? Il 
semble plutôt que le texte copié à la suite de l'Érudition corresponde à la version française des Enseignements de saint Louis à son fils transmise en latin par Robert Gaguin dans son Compendium ${ }^{24}$.

7 Suzanne de Bourbon est l'objet de toutes les attentions didactiques. Au discours maternel qui formait une jeune princesse de quatorze ans bientôt mariée, se substitue le discours plus solennel du docteur en théologie soucieux d'aviver la vigilance d'une future mère. Dans les deux cas, le texte est produit à un moment où la vie de la princesse va connaître une modification profonde: le mariage et la maternité. De nombreux points communs rapprochent les deux ouvrages. Anticipant des maternités à venir, Anne de France signalait déjà à sa fille l'importance de bien éduquer les enfants et lui dispensait par avance quelques conseils ${ }^{25}$. D'autre part, le rôle de mère que s'apprête à endosser Suzanne est une occasion supplémentaire pour la princesse de manifester ses vertus et de faire resplendir sa bonne réputation. Pierre Martin engage Suzanne à « ensuyvre et ymiter les meurs et vertus » d'Anne de France ${ }^{26}$. Renvoyée au prestigieux exemple maternel comme aux faits de ses ancêtres, Suzanne de Bourbon doit perpétuer une tradition qui fait de la bonne éducation une caractéristique de la maison de Bourbon.

La belle éducation bourbonnaise

8 L'éducation des enfants par les parents s'appuie non seulement sur des précédents bibliques mais aussi sur des exemples historiques. S'adressant à Popillon, Pierre Martin rappelle, en se référant à Valère Maxime, que les Romains et les Lacédémoniens accordaient beaucoup d'importance à la formation de leur jeunesse ${ }^{27}$. À cet égard, la réputation de la maison de Bourbon n'est plus à faire car « est rumeur commune que la maison de Bourbon est une des singulieres de toute chretienté pour bien norrir et instruire en bonnes meurs, vertus et devocion, pudicité et toute honnesteté, les enfans des nobles, soient hommes ou femmes. Car c'est une escolle de vertu et de perfection et ou plusieurs nobles desirent leurs enfans estre norriz $»^{28}$. Fille et sœur de roi, régente, duchesse de Bourbon, Anne de France reçut la charge de veiller sur plusieurs jeunes nobles parfois dès leur plus tendre enfance ${ }^{29}$. Louis XI qui voyait en sa fille la «moins folle femme de France $»^{30}$ avait remis à Anne et Pierre la garde du futur Charles VIII. À la mort de sa mère Marguerite de Bourbon, Louise de Savoie, âgée de sept ans, est envoyée avec son petit frère Philibert auprès du duc et de la duchesse de Bourbon ${ }^{31}$. La fillette Marguerite d'Autriche, promise à Charles VIII, est elle aussi confiée à Anne et Pierre. À Amboise, sous la surveillance de la gouvernante Madame de Segré, Marguerite fait son apprentissage de reine ${ }^{32}$. À Moulins, Anne de France assure l'éducation de son futur gendre Charles de Montpensier ${ }^{33}$. Diane de Poitiers, orpheline de mère, est également confiée à la cour bourbonnaise ${ }^{34}$. Aussi lorsque Louis XII se marie avec la jeune Marie d'Angleterre en 1514 demande-t-il à Anne de lui enseigner les «modes et façons de France $»^{35}$. Le compliment de Pierre Martin est confirmé par Brantôme dont la grand-mère avait été élevée avec Anne de France, la «tutrice de roy ", "toujours accompaignée de grand'quantité de Dames et de filles qu'elle nourrissoit fort vertueusement et sagement $\aleph^{36}$. Sous sa conduite, la maison de Bourbon fut un centre prisé par la noblesse : « n'y a guieres heu Dames et filles de grand'maison de son temps qui n'ayt appris leçon d'elle, estant allors la maison de Bourbon l'une des grandes et splendides de la Chrestienté ${ }^{37}$.

9 Surveillante attentive de la noble jeunesse du royaume, Anne de France conseille à Suzanne d'en faire autant avec sa descendance le moment venu car « en ce monde, n'a 
telle joye au père et à la mère, que avoir enfans saiges et bien endoctrinez ». De son côté, Pierre Martin donne plusieurs exemples de bons parents. Outre Anne de France célébrée dès le prologue, Blanche de Castille, la mère des sept Maccabées, ou Monique, mère de saint Augustin, ont veillé au bon comportement de leurs enfants ${ }^{38}$. Dans l'Éthique, Aristote affirme par ailleurs que le rôle du père est essentiel pour l'enfant car « il est cause de son estre et existence par generacion, de sa norriture et permanence par education, de sa discipline et doctrine par informacion $»^{39}$. David, Tobie, Auguste, Louis VIII et le duc Charles $\mathrm{I}^{\mathrm{er}}$ de Bourbon furent de bons pères ${ }^{40}$. Marié à Agnès de Bourgogne, le grand-père de Suzanne eut à cœur de bien endoctriner quatre de ses enfants "en la langue latine laquelle ilz parloient et entendoient». Pierre Martin rappelle leurs brillantes carrières, signes d'une éducation réussie. « Ce furent monsieur l'arcevesque de Lion cardinal, et l'evesque et seigneur du Liege, et deux autres qui succederent a la duché apres luy, monsieur le duc Jehan duc magnanime, lequel luy estant connestable de France, respondoit en bon latin et congreu es ambassadeurs et aux orateurs des universités, et monsieur le bon duc Pierre prince de paix, lequel estoit grant amateur de science et de la parolle de Dieu fondee en bonne doctrine ${ }^{41}$. À charge pour Suzanne et Charles III de faire aussi bien qu'Agnès et Charles I ${ }^{\text {er }}$.

L'éducation des enfants nobles dont la maison de Bourbon se fait une spécialité doit commencer très tôt car "comme la cyre molle reçoit impression, jeunesse prent salutaire amonition et bonne doctrine, et ligerement sans grande dificulté, comme la petite verge et nouvelle plante, on la peult courber, ployer et gouverner $»^{42}$. L'argument est classique ${ }^{43}$. Pierre Martin met par écrit ses recommandations dès la grossesse de Suzanne: ainsi l'éducation de l'héritier ne subira-t-elle aucun délai. Plus facile à modeler, l'enfant est aussi plus exposé en raison de son inexpérience. Les parents doivent pallier cette faiblesse qui perdure à l'adolescence. Consciente des fragilités de Suzanne, une jeune fille prête à être mariée en 1505 , et poussée par une "parfaicte amour naturelle", Anne de France se propose de rassembler "aucuns petis enseignemens, advertissans vostre ignorance et petite jeunesse ». En 1517 on attend de Suzanne qu'elle puisse à son tour donner le bon exemple à ses enfants car « la faulte des bonnes meurs des enfans provient souvent de la maulvaise vie et exemplarité des parentz ». Des enfants mal éduqués trahissent de mauvais parents. Devenue mère, la noble dame met donc en jeu ce qu'elle a de plus cher : son honneur. Avertie de longue date, Suzanne connaît la fragilité de ce bien car « ne peult estre si peu quassé ou effacé, que jamais on y trouve réparation digne à y satisfaire $»^{44}$. L'éducation de l'enfant noble incombe aux parents dont elle révèle les qualités et les défauts.

11 Au-delà des conséquences personnelles, l'éducation de la jeunesse est primordiale pour la chose publique chère à Pierre Martin. Les princes mal éduqués peinent à conduire correctement leurs sujets car « ilz ne peuvent delaisser ne oblier les meurs et manieres de vivre de jeunesse ». Dans ce cas, point de bon gouvernement: «en lieu de justice font injustice, en lieu de garder leurs subgectz les oppriment, et en lieu de leur monstrer bonne exemple les scandalizent». Pour le frère prêcheur, une bonne éducation contribue à la continuelle prospérité de la maison princière. Aussi ceux qui désobéissent à Dieu ou manquent de respect à l'Église et aux clercs perdent-ils leur royaume. Les exemples de Saul et Pompée sont là-dessus très clairs ${ }^{45}$.

12 Salut de l'enfant, honneur des parents, efficacité du gouvernement, les enjeux de l'éducation des enfants et des adolescents nobles sont multiples. Anne de France écrit pour former sa fille tandis que Pierre Martin écrit pour aider à l'éducation d'un héritier 
dont le sexe n'est pas encore connu. Il attend sans doute avec impatience la naissance d'un petit garçon. Les exemples du Traité de l'érudition et de l'enseignement des enfants des nobles sont souvent masculins mais pas exclusivement. Les filles y ont une petite place. Et les deux textes se rejoignent sur bien des points. «Noblesse, tant soit grande, ne vault riens, si elle n'est aournée de vertus ", déclare Anne à Suzanne. En 1517 Pierre Martin ne lui dit pas autre chose: "c'est chose plus louable et digne d'honneur noblesse de vertus que de sang, et ne vault gueres et est peu a priser noblesse de sang sans noblesse de vertu, et qui loue ou honnore aucuns pour leur noble generation y loue leurs predecesseurs et non pas eulx $»^{46}$. La noblesse doit être vertueuse ${ }^{47}$. La base de toute éducation, sur laquelle Anne de France et Pierre Martin s'accordent, est morale.

La formation de la noblesse : quelques aspects

13 Pour définir les règles de vie nécessaires à une noblesse vertueuse digne de la maison de Bourbon, Anne de France et Pierre Martin puisent dans des sources qui réservent peu de surprises. Anne de France se sert de son expérience personnelle et cite les Pères et Docteurs de l'Église, Boèce et le « docteur Lyénard » très souvent, Socrate, Ovide et Aristote ponctuellement ${ }^{48}$. Docteur en théologie, Pierre Martin compile les citations des Écritures. Chaque chapitre de l'Érudition ou presque commence par les Psaumes de David qui sont une référence récurrente dans le traité. Il a recours également à de nombreux auteurs antiques dont Aristote, Cicéron, Sénèque, Salluste, Plutarque ou encore Végèce ${ }^{49}$. Enfin il est ouvert aux auteurs plus récents comme Jean Gerson, Robert Holcot ou Antonin de Florence ${ }^{50}$.

14 L'éducation religieuse est essentielle. Anne de France résume les principaux points auxquels Suzanne devra être attentive avec ses enfants. «Par quoy n'y devez plaindre vostre peine, à les bien enseigner et aprendre, selon vostre pouvoir, et leur petit entendement, premièrement les articles de la foy, les commandements de la loy, et en quelle manière on y peult pécher ; aussi des sept péchez mortelz, et comment on se doit confesser, leurs contenances à l'église et aux prédications, et comment en grant révérence et humilité de cueur, doivent recevoir leur créateur ». Il faut surveiller particulièrement les filles car "c'est charge bien dangereuse $»^{51}$. Sur le modèle de sainte Anne, la mère est une enseignante avisée grâce aux livres d'heures qui servent aussi de premiers livres de lecture aux enfants ${ }^{52}$. Anne de France préconise de prier souvent et de suivre la messe « en grant dévotion et tous jours à genoulx, si possible est, en aïant les yeulx ententiz envers le prestre à l'autel, ou en vostre livre ». Pierre Martin développe le programme proposé par Anne de France sans innover. Le noble vertueux est d'abord un bon chrétien. Le frère y consacre l'essentiel du chapitre cinq. Dès l'enfance, les parents doivent inculquer les bases de la foi, la façon de se tenir à l'église, la nécessité de se confesser et d'écouter avec attention la parole divine ${ }^{53}$. Outre les gestes de dévotion à accomplir, il faut quotidiennement rechercher les vertus et fuir les vices. Orgueil, luxure, gloutonnerie et ivresse sont des écueils à éviter. Ils sont souvent favorisés par l'oisiveté qui encourage à mal agir. Pierre Martin consacre de longs chapitres du traité à ces thèmes ${ }^{54}$.

Plongé dans le monde, le noble doit être vigilant envers son entourage. De sa mère, Suzanne apprend la bonne façon de se comporter avec les autres et à demeurer toujours méfiante car «dient les saiges que on doit avoir yeulx pour toutes choses regarder, et rien veoir, oreilles pour tout ouyr et rien sçavoir, langue pour respondre à chascun, sans dire mot qui à nully puisse estre en rien préjudiciable». Pierre Martin 
insiste sur le danger que représentent les flatteurs et les moqueurs ${ }^{55}$. Mais la vigilance doit aussi s'exercer envers soi-même. Le contrôle des gestes est généreusement et classiquement traité par Anne de France car le regard des hommes sur le comportement féminin est inquisiteur, particulièrement envers les nobles dames qui doivent représenter un idéal de perfection. Aussi Anne met-elle en garde : « ne faictes de vos mains, comme font aucunes jeunes filles, qui, par folle acoustumance, ont tousjours sans cause la main au nez, ou à la bouche, aux yeulx, ou aux oreilles, qui est très mal séant, mesmement à nobles femmes, qui, voluntiers, sont plus regardées que les autres [...] car, en toutes choses, elles sont, et doivent estre, le mirœr patron et exemple des autres». Pierre Martin s'intéresse aussi à la discipline de l'apparence car « veoir un gentilhomme bigarré et dechiqueté n'est pas signe de prudence ou gravité, mais de follie et ligereté, et peult on juger qu'ilz sont autant descoupez, dechiquetés et variez par dedans en leur esprit comme par dehors $»^{56}$. Une apparence désordonnée trahit une disposition intérieure incontrôlée et vicieuse. En s'appuyant sur saint Paul, Pierre Martin souligne l'importance pour les femmes nobles d'avoir un habillement honnête. Là encore Suzanne savait déjà, grâce à sa mère, qu'il lui faudrait tenir ses filles " raisonnablement habillées ${ }^{57}$.

La formation intellectuelle est envisagée également dans les deux textes. Anne de France conseille rapidement à Suzanne quelques lectures édifiantes comme le « livret du preudhomme de sainct Lis, celui de sainct Pierre de Luxembourg, les sommes le roy, l'orologe de Sapience, ou aultres livres de vie des Saincts, aussi les dictz des philosophes et anciens saiges » qui permettent de passer le temps de façon honnête ainsi que la fréquentation des sages "pour apprendre et retenir quelques bons enseignemens et doctrines $»^{58}$. Pensant à la naissance prochaine d'un héritier mâle, Pierre Martin proclame que "c'est chose vituperable et deshonneste d'estre noble homme imprudent, ignorant et mal instruit ». Il recommande le choix d'un précepteur compétent car «si les princes et nobles mectent doncques grande diligence de chercher et trouver bonne norrice aiant bon laict pour bien norrir le corps de leurs enfans, par plus forte et meilleure raison doibvent labourer et mectre peine de pourveoir d'ung bon et scientificque pere norricier, c'est a dire d'ung bon maistre et precepteur aiant bon laict de bonnes meurs et de science pour norrir et endoctriner l'ame de leur lignee ». Sur le modèle de Plutarque pour Trajan, Sénèque pour Néron, Aristote pour Alexandre, Alcuin pour Charlemagne, le précepteur saura ouvrir l'esprit de son élève et, "selon l'eage ", lui faire étudier les arts libéraux, les sciences humaines et les histoires «bonnes, honnestes, pudicques et catholicques ${ }^{59}$. Pierre Martin n'oublie pas de signaler que les frères mendiants sont de très bons maîtres. Louis VIII et Blanche de Castille ne s'y sont pas trompés puisque « les parentz du roy sainct Loys, pour le faire bon et scientificque, luy donnerent pour maistres et precepteurs deux bons docteurs en theologie, l'ung de l'ordre des freres prescheurs et l'autre de l'ordre des freres mineurs $»^{60}$. Remarque intéressante car on ignore qui a pris en charge la formation du saint roi $^{61}$. Mais Pierre Martin est certain que Louis IX a reçu un enseignement de grande qualité évidemment dispensé par les ordres mendiants. Peut-être plaide-t-il indirectement pour qu'on lui confie ce rôle auprès du futur héritier?

Pierre Martin partage bien des points communs avec ses prédécesseurs qui ont écrit pour les rois et les princes. Il suit les traces d'autres prêcheurs comme Guillaume Peyraut ou Vincent de Beauvais qui adressa son traité à la reine Marguerite de Provence pour aider à l'éducation des enfants royaux. Originaire de Bourges, Pierre Martin a pu aussi apprécier le traité composé par Gilles de Rome, archevêque de cette 
ville à partir de 1295. Les prescriptions de Pierre Martin recoupent en partie celles dispensées par Anne de France et par ces textes bien connus ${ }^{62}$. Au début du XVI $\mathrm{X}^{\mathrm{e}}$ siècle, l'intérêt que l'on porte aux anciens miroirs des princes est notable. La traduction en français du Liber de informatione principum faite par Jean Golein pour le roi Charles V est éditée à Paris en 1517. Le Speculum dominarum écrit par Durand de Champagne pour la reine Jeanne de Navarre $(\dagger 1305)$ est traduit et remanié par Ysambert de Saint-Léger pour la sœur de François I ${ }^{\mathrm{er}}$, Marguerite de Navarre, vers $1528^{63}$. À défaut d'être originale dans ses préceptes, l'Érudition de Pierre Martin se veut bourbonnaise dans ses exemples.

Pierre Martin et les Bourbon

18 L'histoire est riche de rois prestigieux, bibliques ou historiques, comme Charlemagne et Robert le Pieux. Mais le frère valorise également des exemples destinés à célébrer la gloire propre des Bourbon comme le duc Charles $\mathrm{I}^{\mathrm{er}}$ et ses fils déjà mentionnés ou encore Louis IX et Louis II de Bourbon, le saint roi et le bon duc, car «c'est ung beau tiltre pour ung prince d'estre appellé bon ou sainct $»^{64}$.

Les ducs de Bourbon sont issus de Louis IX par Robert de Clermont son sixième fils. Ils sont de sang royal et aiment à souligner cette prestigieuse filiation. Le cardinal Charles de Bourbon qui commande dans les années 1480 un Livre des faiz Monseigneur saint Loys à la demande d'une duchesse de Bourbon non identifiée admire le saint roi. La miniature de dédicace le présente entouré des statues de Charlemagne et saint Louis. À sa mort, le cardinal est enterré dans une chasuble portant l'emblème de l'ordre de la Cosse de Genêt fondé, selon la tradition, par Louis IX en $1234^{65}$. L'admiration se manifeste aussi par la construction de saintes-chapelles sur le modèle royal parisien. En 1315, Louis I ${ }^{\mathrm{er}}$ de Bourbon fonde la première sainte-chapelle de Bourbon l'Archambault et en 1483 le duc Jean II y fait construire une seconde sainte-chapelle dédiée à saint Louis ${ }^{66}$. Ainsi Pierre Martin rappelle à Suzanne ses prestigieux prédécesseurs «en commancent depuis le bon roy sainct Loys " dont l'exemple est utilisé à plusieurs reprises dans le traité pour illustrer le thème du roi dévot et savant aux côtés de Robert le Pieux et de Charlemagne ${ }^{67}$.

Le duc Louis II, surnommé «le Bon», fait lui aussi rayonner la gloire des Bourbon ${ }^{68}$. Otage à la place du roi Jean, fondateur de l'ordre de l'Écu d'or vers 1367, Louis II participe à la conquête du Poitou et de la Guyenne et à la bataille de Roosebecke en 1382 sous Charles VI. C'est un modèle de prince chrétien qui remporte face aux Infidèles " une victoire grande et digne de memoire ${ }^{69}$ et fonde les chanoines de NotreDame de Moulins et les Célestins de Vichy. Là encore, la maison de Bourbon célèbre depuis longtemps cet ancêtre puisque sur l'ordre de Charles de Bourbon, comte de Clermont, Jean Cabaret d'Orville dès 1429 avait mis par écrit la vie de Louis II réputé pour sa valeur guerrière, sa piété et sa sagesse ${ }^{70}$.

21 En 1505, Anne de France conseillait à sa fille de ne pas mépriser ses ancêtres ${ }^{71}$. En 1517, Pierre Martin met l'accent sur le prestige bourbonnais. Est-ce un effet de l'avènement des Angoulême au trône ? À la tête d'une grande principauté, le duc Charles III est fait connétable par François $\mathrm{I}^{\mathrm{er}}$ : le plus puissant vassal du roi détient l'office le plus important du royaume ${ }^{72}$. En 1517, alors que les relations avec le roi sont tendues, la naissance prochaine d'un héritier, peut-être masculin, vient conforter l'avenir de la maison ducale de Bourbon et alimenter une certaine concurrence d'autant que la mère de François ${ }^{\text {er }}$, Louise de Savoie, est la cousine germaine de Suzanne de Bourbon ${ }^{73}$. Alors que l'Occident pense à nouveau à la croisade, tant les Angoulême que les Bourbon 
peuvent incarner l'idéal du prince chrétien. En 1515 François I ${ }^{\mathrm{er}}$ rencontre le pape Léon $\mathrm{X}$ à Bologne et reçoit un reliquaire contenant un fragment de la Vraie Croix ${ }^{74}$. Les ducs de Bourbon ont, quant à eux, régulièrement prouvé leur attachement à l'idée de croisade. Le premier duc, petit-fils de saint Louis, faisait vœu de se croiser dès 1316 . Pierre $\mathrm{I}^{\text {er }}$ participe à la croisade de Prusse en 1344-1345 ${ }^{75}$. En 1517, alors que François I ${ }^{\text {er }}$ est comparé à Constantin, Pierre Martin rappelle dans son traité que Louis II de Bourbon a affronté les Infidèles lors de la croisade de Barbarie en 1390. Mais le frère reste prudent. Tout en renvoyant au passé prestigieux des ducs, il dénonce longuement le péché d'ambition car "non obstant que toute espece d'orgueil soit dangereuse et maulvaise, toutesfois entre les aultres les plus dangereuses et perilleuses es princes sont ambition, vaine gloire et inobeissance a Dieu et a leurs superieurs ${ }^{76}$.

Revenant de l'entrée royale à Rouen en juillet $1517^{77}$, le duc Charles III de Bourbon assiste à la naissance de son fils, le premier mâle bourbonnais depuis longtemps « car en ladite maison de Bourbon n'y avoit point eu de fils depuis les enfans du duc Charles $\mathrm{I}^{\mathrm{er}}$, quatre-vingts ans avoit ». Le baptême fut célébré par l'évêque de Lisieux dans la chapelle du château de Moulins. L'enfant prend le titre de comte de Clermont et les fastes durèrent «l'espace de douze ou quinze jours, le tout ès dépens de mondit sieur de Bourbon ${ }^{78}$. Dans ses Enseignements, Anne de France recommandait à Suzanne de choisir avec soin les parrains et marraines de ses enfants. «Ceux-là doivent estre saiges et de honnestes condicions, sans faire comme font aucuns, à qui il ne chault qui baptise ou tienne leurs enfants $»^{79}$. C'est chose faite en 1517. En faisant porter le bébé sur les fonts baptismaux par François $I^{e r}$, Suzanne et Charles font du roi de France le père spirituel de leur fils. Le nouveau-né prend le prénom de son parrain ${ }^{80}$ et aurait été armé chevalier par Bayard comme lui ${ }^{81}$. Les liens distendus sont ainsi renforcés par des liens symboliques $^{82}$. Reste à faire du fils spirituel du roi un prince vertueux et bien éduqué. Louise de Savoie fut le véritable " compas et mesure " de François d'Angoulême ${ }^{83}$. Grâce à Pierre Martin et son traité, Suzanne de Bourbon espère devenir à son tour le compas de son enfant. Le décès précoce de François de Bourbon en 1518 sonne le glas des aspirations bourbonnaises. À la mort de Suzanne en 1521, la principauté s'effondre ${ }^{84}$.

"Escolle de vertu et de perfection », l'éloge de Pierre Martin s'applique à la maison de Bourbon au début $\mathrm{du} \mathrm{XvI}^{\mathrm{e}}$ siècle sous l'effet d'une double nécessité. On attendait beaucoup de Suzanne car à court terme l'avenir des ducs reposait en partie sur ses frêles épaules. Mais la fille d'Anne de France devait aussi être digne d'une ascendance remarquable par ses vertus et sa sagesse. Les traités écrits pour cette princesse se rattachent à la féconde tradition didactique de la famille royale à laquelle la maison de Bourbon est liée. Anne de France et Pierre Martin sont les lointains et respectueux héritiers de Louis IX, Vincent de Beauvais ou Gilles de Rome. Comme ses aïeux, comme son père, Anne de France veille à l'éducation des jeunes qui l'entourent ${ }^{85}$. À son tour Suzanne endosse cette responsabilité. Comme d'autres mendiants, Pierre Martin conseille la haute noblesse grâce aux cas édifiants laissés par l'histoire biblique, antique et nationale ${ }^{86}$. Par leur excellence, les princes et les princesses de la maison de Bourbon se montrent dignes de leurs ancêtres et contribuent à l'édification d'autrui. Le miroir qu'on leur tend les corrige et renvoie aux autres leur image parfaite ${ }^{87}$. 


\section{NOTES}

1. J. KRYNEN, L'Empire du roi. Idées et croyances politiques en France, $\mathrm{XIII}^{e}$-XVe siècle, Paris, 1993, p. 168 et suiv. Voir L. K. BoRN, « The Perfect Prince : A Study in Thirteenth and Fourteenth-Century Ideals ", Speculum, 1928, III, p. 470-504. D. M. BELL, L'idéal éthique de la royauté en France au Moyen Âge d'après quelques moralistes de ce temps, Paris, 1962. R. HALÉVI (dir.), Le savoir du prince, du Moyen Âge aux Lumières, Paris, 2002. M. SENELLART, Les arts de gouverner. Du regimen médiéval au concept de gouvernement, Paris, 1995.

2. Vincent De BeAuvais, De eruditione filiorum nobilium, A. STEINER éd., Cambridge, 1938.

3. Li livres du gouvernement des rois, S. P. MOLENAER éd., New York, 1899 (traduction d'Henri de Gauchy).

4. Le livre du chevalier de la Tour Landry pour l'enseignement de ses filles, A. MONTAIGLON éd., Paris, 1854. Cf. A.-M. DE GENDT, L'Art d'éduquer les nobles damoiselles. Le livre du chevalier de la Tour Landry, Paris, 2003, et " Aucuns petis enseignemens : "Home-made" Courtesy Books in Medieval France ", dans Centres of Learning. Learning and Location in the Pre-Modern Europe and the Near East, J. W. DRIJVERS et A. A. MACDonALD éd., Leiden, 1995, p. 279-288.

5. D. O'ConNell, Les propos de Saint Louis, Paris, 1974.

6. DHUODA, Manuel pour mon fils, P. RichÉ éd., B. DE VREGILLE et C. MONDÉSERT trad., Paris, rééd. 1991.

7. M. ZINK, La prédication en langue romane avant 1300, Paris, 1976, p. 152. Sachiez certainement, Bibliothèque Mazarine, ms 788, fos 79-84.

8. A. HeNTSCH, De la littérature didactique du Moyen Âge s'adressant spécialement aux femmes, réimpr. Genève, 1975, p. 135. Un exemplaire de l'ouvrage d'Élisabeth de Bosnie († 1382), épouse du roi Louis $\mathrm{I}^{\mathrm{er}}$ de Hongrie et mère de trois filles, aurait été remis à Louis de France, comte de Valois en 1374. Cf. A.-M. DE GENDT, « Le Livre de la royne Prines : le modèle qui n'existe pas? ", L'Art d'éduquer, op. cit., p. 245-252.

9. J. BOUCHET, Le Panégyrique du chevalier sans reproche, ou Mémoires de La Trémouille, Collection des Mémoires relatifs à l'Histoire de France, Paris, 1820, p. 128. G. DE BourBon, CEuvres spirituelles, 1510-1516, É. BERRIOT-SALVADORE éd., Paris, 1999, p. 8-9 et p. 37. A. HENTSCH, op. cit., p. 207.

10. Suzanne de Bourbon est née le 10 mai 1491 et mariée à son cousin Charles le 10 mai 1505. Elle meurt le 28 avril 1521. P. ANSELME, Histoire généalogique et chronologique de la maison royale de France, Paris, 1726, t. 1, p. 313 et p. 317.

11. En 1503, Symphorien Champier adresse à Suzanne le livre deux de la Nef des dames vertueuses intitulé le « gouvernement de mariage ». Les autres livres de la Nef sont dédiés à Anne de France.

12. Les Enseignements d'Anne de France duchesse de Bourbonnois et d'Auvergne à sa fille Susanne de Bourbon, A.-M. CHAZAud éd., Moulins, 1878, réimpr. Marseille, 1978 [Enseignements]. Sur le feuillet de garde du manuscrit de Saint-Pétersbourg édité (planche 1) : «Se livre est à moy Susanne de Bourbon, et l'ey eu de la mezon de Borbon ». Le texte est écrit entre la mort de Pierre II et le mariage de Suzanne. R. L. KRUEGER, « Chascune selon son estat: Women's Education and Social Class in Conduct's Books of Christine de Pizan and Anne de France ", Papers on French Seventeenth Century Literature, XXIV, 1997, $\mathrm{n}^{\circ} 46$, p. 19-34. 
13. G. DE MARILLAC, Vie du connétable Charles de Bourbon, de 1490 à 1521 par Guillaume de Marillac son secrétaire, dans Choix de chroniques et mémoires relatifs à l'histoire de France, J.A. C. Buchon éd., Orléans, 1875, p. 167.

14. Pierre Martin entre au couvent des Jacobins de Bourges dont il devient le prieur. Docteur en théologie, il enseigne à l'université de Bourges. Confesseur d'Anne, provincial de France et inquisiteur en 1518, il meurt le 25 décembre 1527. J. QUETIF et J. ECHARD, Scriptores ordinis praedicatorum, II, p. 72-73. A. MENU, Monographie du couvent des Jacobins de Bourges, Bourges, 1873, p. 94-96. R. SAUZET, Les réguliers mendiants acteurs du changement religieux dans le royaume de France, 1480-1560, Tours, 1994, p. 162. Dictionnaire des lettres françaises. Le XVI ${ }^{e}$ siècle, Paris, 2001, p. 813. H. MARTIN (Le métier de prédicateur à la fin du Moyen Âge, Paris, 1988, p. 677) répertorie un frère prêcheur Pierre Martin (homonyme ?) à Amiens en 1511.

15. Ms. Sainte-Geneviève 2221, 78 feuillets en parchemin, [Érudition]. Le traité occupe les fos 2-72. C. KOHLER, Catalogue des manuscrits de la Bibliothèque Sainte-Geneviève, t. 2 , Paris, 1896, p. 291-2.

16. Érudition, fos 2-3. Gradué de l'université, fils de Charles Popillon qui présida la Chambre des comptes de Moulins de 1487 à 1507, Pierre Popillon épouse Claude Herbelot. Trésorier, argentier et maître de la Chambre aux deniers de Suzanne de Bourbon, il devient maître des requêtes de Pierre II puis notaire et secrétaire du roi. Chancelier de Bourbon de 1515 à 1523, à la tête de nombreuses seigneuries, Pierre Popillon est témoin lors du testament de Suzanne et lors de la donation faite par Anne à son gendre. Inquiété après les déboires du connétable, il meurt emprisonné à la Bastille en août 1524. O. MATTÉONI, Servir le prince. Les officiers des ducs de Bourbon à la fin du Moyen Âge, 1356-1523, Paris, 1998, p. 142, 416 et 432. J.-M. DE LA MURE, Histoire des ducs de Bourbon et des comtes de Forez, Paris, 1868, II, notes p. 556, 558 et 620.

17. Érudition, fo $2 v^{\circ}$.

18. Érudition, $\mathrm{f}^{\mathrm{o}} 4 \mathrm{v}^{\mathrm{o}}$. Allusion également à la grossesse de Suzanne dans la lettre à Pierre Popillon, fo 3.

19. Érudition, fo $70 \mathrm{v}^{\circ}$. Une table des matières récapitule l'ensemble, fos $71 \mathrm{v}^{\mathrm{o}}-72$.

20. Érudition, fo $78 \mathrm{v}^{\circ}$. La date de naissance copiée dans le manuscrit diffère de celle donnée par d'autres sources en juillet 1517 (G. DE MARILLAC, op. cit., p. 168 et J.-M. DE LA MuRE, op. cit., p. 550).

21. Érudition, fo $78 \mathrm{~V}^{\circ}$. Ps. 85.

22. Érudition, fos $73-73 v^{\circ}$. Le titre « La forme et maniere que Dieu revella a Moyse de donner la benediction es enfants » est suivi de la citation latine de Num. 6, 24-26. Cf. Postillae super totam Bibliam, N. DE LYRE, Biblie jampridem renovate pars prima, cum additionibus Burgensis ac replicis Thoringi, Basileae, 1502, vol. 1, fo $275 \mathrm{v}^{\circ}$, cap. 6. Sur Nicolas de Lyre, voir P. Buc, « The Book of Kings : Nicholas of Lyra's mirror of Princes », dans Nicholas of Lyra. The Senses of Scripture, P. D. W. KREY et L. SMITH éd., Leiden-BostonCologne, 2000, p. 83-109 ; « Pouvoir royal et commentaires de la Bible (1150-1350) », Annales ESC, 1989, n 3, p. 691-713.

23. Érudition, fos $74-78$.

24. Érudition, fo 74 . Le texte conservé à Sainte-Geneviève date la découverte de Gérard de Montaigu de 1364 (au lieu de 1374) comme Gaguin et ne dit mot de l'obéissance due au pape. R. GAGUIN, Compendium Roberti Gaguini super Francorum gestis, ab ipso recognitum et auctum, 1500, liber VII, fos LXIII-LXIIII, Regis divi Ludovici ad filium precepta. Cf. H. F. DELABORDE, « Le texte primitif des enseignements de saint Louis à son fils ", Bibliothèque 
de l'École des Chartes, 1912, p. 73-100 et surtout p. 91. F. COLLARD, Un historien au travail à la fin du Xve siècle : Robert Gaguin, Genève, 1996, p. 150-151 et p. 272.

25. Champier aborde aussi l'éducation des enfants dans le chapitre 19 du livre 2 de sa Nef (cf. note 11).

26. Érudition, $\mathrm{f}^{\circ} 4 \mathrm{v}^{\circ}$.

27. Érudition, $\mathrm{f}^{\circ \mathrm{s}} 2-2 \mathrm{v}^{\circ}$. Exemple des Anciens qui confiaient l'éducation des fils des grandes familles aux peuples d'Étrurie. Cf. VALÈre MAXIME, Faits et dits mémorables, R. COMBes éd., Paris, 1995, t. 1, livre 1, p. 100.

28. Érudition, $\mathrm{f}^{\circ} 4 \mathrm{v}^{\circ}$ (prologue adressé à Suzanne).

29. E. VIENNOT, « La transmission du savoir-faire politique entre femmes, d'Anne de France à Marguerite de Valois », dans La Transmission du savoir dans l'Europe des $\mathrm{XVI}^{e}$ et XVII ${ }^{e}$ siècles, M. RoIG MIRANDA dir., Paris, 2000, p. 87-98.

30. J. FAVIER, Louis XI, Paris, 2001, p. 901.

31. P. HENRY-BORDEAUX, Louise de Savoie, « roi » de France, Paris, rééd. 1971, p. 24-27.

32. F. TROMBERT, « Une reine de quatre ans à la cour de France : Marguerite d'Autriche, 1484-1485 ", dans Autour de Marguerite d'Écosse. Reines, princesses et dames du Xve siècle, G. et P. ContAminE dir., Paris, 1999, p. 123-161. G. DE Boom, Marguerite d'Autriche-Savoie et la Pré-Renaissance, Paris, 1935, p. 10-12. C. C. WILLARD, « Anne de France, reader of Christine de Pizan ", dans The Reception of Christine de Pizan from the fifteenth through the nineteenth centuries. Visitors to the City, G. K. McLEOD dir., 1991, p. 59-70.

33. G. DE MARILLAC, op. cit., p. 132 : «Bien faisoit-elle nourrir et entretenir ledit comte Charles, luy faisant apprendre le latin à certaines heures du jour et quelquesfois à courir la lance, piquer les chevaux, tirer de l'arc où il étoit enclin; autrefois aller à la chasse ou à la volerie, et aussi en tous autres déduits et passe-temps où l'on a accoutumé d'induire les grands seigneurs ». La sœur de Charles, Louise de Bourbon, a également été « nourrie dès son enfance et mariée » par Anne de France.

34. I. Cloulas, Diane de Poitiers, Paris, 1997, p. 21.

35. P. PRADEL, Anne de France, 1461-1522, Paris, 1986, p. 197.

36. Louise de Daillon du Lude, femme d'André de Vivonne, sénéchal de Poitou.

37. BRANTÔME, Recueil des Dames, poésies et tombeaux, E. VAUCHERET éd., Paris, 1991, Recueil des Dames, I, VI : « Discours sur mesdames, filles de la noble maison de France ", p. 167 et suivantes.

38. Enseignements, p. 104. Érudition, fos $6 \mathrm{v}^{0}-7:$ mère des Maccabées (II Mac. 7).

39. Érudition, $\mathrm{f}^{\circ} 6 \mathrm{~V}^{\circ}$. Cf. ARISTOTE, Éthique à Nicomaque, VIII, 13, J. TRICOT éd., Paris, 1994, p. 415.

40. Érudition: David avec Salomon, $\mathrm{f}^{\circ} 7$ et fo $10 \mathrm{v}^{\circ}$ (III Reg. 2 ; I Chron. 28 ; Pro. 4); " Thobie ", fo 7 (Tob. 1 et 4); " Octavien saige empereur », fo 68 . Mais il existe aussi des pères incompétents : David avec Amnon, fo $11 v^{\circ}$ (II Reg. 13) ; Lucrecius, fo 13 (cf. PseUDoBoÈcE, De disciplina scolarium, 2, 8-9, O. WeIJERS éd., Leiden-Köln, 1976, p. 102) ; Éli avec Hophni et Pinhas, fo 14 (I Reg. 2-4). Sur l'image du père, voir D. LETT, « Tendres souverains. Historiographie et histoire des pères au Moyen Âge ", dans Histoire des pères et de la paternité, J. Delumeau dir., Paris, rééd. 2000, p. 17-40 et notamment p. 26-31.

41. Érudition, fos $38 \mathrm{v}^{\circ}-39$. Jean II, époux de Jeanne sœur de Louis XI (1427-1488); Charles II cardinal-archevêque de Lyon (1434-1488); Pierre II époux d'Anne de France (1438-1503); Louis évêque de Liège (1438-1482).

42. Érudition, $\mathrm{f}^{\circ} 6$. Sur l'enfance, voir D. LETT, L'enfant des miracles. Enfance et société au Moyen Âge, Paris, 1997. D. ALEXANDRE-BiDon et D. LETT, Les enfants au Moyen Âge, $\mathrm{V}^{e}$-XV ${ }^{e}$ 
siècles, Paris, 1997, en particulier p. 73-96 et p. 189-218. E. BECCHI et D. JuLIA, Histoire de l'enfance en Occident, t. 1, Paris, 1998. Sur l'éducation des enfants nobles, voir N. ORME, From Childhood to Chivalry. The Education of English Kings and Aristocracy, 1066-1530, London, 1984, et Education and Society in Medieval and Renaissance England, London, 1989. 43. Par exemple, Vincent de Beauvais (éd. A. STEINER, p. 7 et 84), Guillaume Peyraut (De eruditione principum, dans THOMAS AQUINAS, Opera omnia, XVI, opusculum 37, réimpr. New York, 1950, p. 429) et Gilles de Rome (éd. S. P. MOLENAER, p. 196 et 215) ont recours à l'image de la cire ainsi que Christine de Pizan (Le Livre des faits et bonnes mours du roi Charles V le Sage, E. Hicks et T. Moreau éd., Paris, 1997, p. 50).

44. Enseignements, p. 1 et p. 127. Érudition, fo 12. Sur l'honneur féminin, C. GAUVARD, « Honneur de femme et femme d'honneur en France à la fin du Moyen Âge », Francia, 2001, 28/1, p. 159-191.

45. Érudition, $\mathrm{f}^{\mathrm{o}} 13 \mathrm{v}^{\circ}, \mathrm{f}^{\circ \mathrm{s}} 22-22 \mathrm{v}^{\circ}$. Cf. les trois premiers chapitres : « Comment les parens doivent avoir solicitude de bien endoctriner leurs enfans » $\left(f^{\circ} 5 v^{\circ}\right)$; « Les biens qui proviennent de la bonne erudition des enfans des nobles » ( $\left.f^{\circ} 8\right)$; « Les maulx qui adviennent de la negligence de l'erudition des jeunes enfans » ( $\left.f^{\circ} 11\right)$.

46. Enseignements, p. 57. Érudition, fos $14 \mathrm{v}^{\mathrm{o}}-15$.

47. Voir P. Contamine, La noblesse au royaume de France, de Philippe le Bel à Louis XII, Paris, 1997 ; K. F. WERNER, Naissance de la noblesse. L'essor des élites politiques en Europe, Paris, 1998 ; E. SCHALK, L'épée et le sang. Une histoire du concept de noblesse (vers 1500-vers 1650), Paris, 1996 ; J. C. WARD, « Noblewomen, Family, and Identity in Later Medieval Europe », dans Nobles and Nobility in Medieval Europe. Concepts, origins, transformations, A. J. DUGGAN éd., Woodbridge, 2000, p. 246-262.

48. « Le docteur Lyénard » est identifié avec le prêcheur Léonard d'Udine (A. HENTSCH, op. cit., p. 199) ou avec Leonardo Bruni (C. MARTIN-UlRICH, « Bon fruict et deceptable monde : Anne de Beaujeu et l'héritage augustinien », dans Reines et Princesses au Moyen Âge, Cahiers du CRISIMA, no 5, Montpellier, 2001, p. 473-474).

49. Sur Végèce et les miroirs, P. RICHARDOT, Végèce et la culture militaire au Moyen Âge, $\mathrm{V}^{e_{-}}$ $\mathrm{XV}^{e}$ siècles, Paris, 1998, p. 77 et suivantes.

50. Érudition, fo 16 et fo 21 (Jean Gerson) et fo $46 \mathrm{v}^{\circ}$ (Robert Holcot ; Antonin de Florence). Dans son Tractatus destiné au précepteur du dauphin Louis, Jean Gerson fait référence au duc Louis II de Bourbon. Cf. A. ThomAs, Jean de Gerson et l'éducation des dauphins de France, Paris, 1930, p. 43-44.

51. Enseignements, p. 104-106.

52. D. AlEXANDRE-BiDON, « Des femmes de bonne foi. La religion des mères au Moyen Âge ", dans La religion de ma mère. Les femmes et la transmission de la foi, J. DELUMEAU dir., Paris, 1992, p. 91-122.

53. Enseignements, p. 64. Érudition, fos 19-21.

54. Érudition, voir en particulier les chapitres 9-10-11-12.

55. Enseignements, p. 20. Érudition, fo 25.

56. Enseignements, p. 64. Érudition, fo 31.

57. Enseignements, p. 106. Érudition, fos $31 \mathrm{v}^{\mathrm{o}}-32$ (I Tim. 2). Sur la critique de la mode, M.G. MuZZARELLI, Gli inganni delle apparenze. Disciplina di vesti e ornamenti alla fine del Medioevo, Turin, 1996. D. OWEN-HugHES, «Les modes ", dans Histoire des femmes en Occident, t. 2, C. KLAPISCH-ZUBER dir., Paris, rééd. 2002, p. 181-208.

58. Enseignements, p. 8 et p. 124. On identifie l'ouvrage de Jean de Varennes, ermite de Saint-Lié (A. VAUCHEZ, « Un réformateur religieux dans la France de Charles VI : Jean de 
Varennes, († 1396 ?) », Académie des Inscriptions et Belles-Lettres - Comptes-rendus des séances, 1998, IV, p. 1111-1128 : la note 6 signale la découverte par Geneviève Hasenohr de deux œuvres spirituelles de Jean de Varennes, l'Épître du miroir de chrétienté</I > et la $<$ I>Médecine de l'âme en l'article de la mort) ; le Livret du cardinal Pierre de Luxembourg († 1387) ; la Somme le Roi de frère Laurent (1279) ; l'Horologium sapientiae du frère prêcheur Henri Suso ( $†$ 1366) traduit vers 1389 par un frère mineur de Neufchâteau (J. ANCELET-HustACHE, « Quelques indications sur les manuscrits de l'Horloge de Sapience ", Heinrich Seuse. Studien, Köln, 1966, p. 161-170 ; H. SuSo, Euvres complètes, J. ANCELET-HuSTACHE éd., Paris, 1977) ; peut-être La Légende dorée de Jacques de Voragine et les Dits moraux des philosophes traduits par Guillaume de Tignonville.

59. Érudition, fos $37-39 \mathrm{v}^{\circ}$.

60. Érudition, fo $38 \mathrm{v}^{\circ}$.

61. J. RICHARD, Saint Louis, Paris, 1983, p. 31.

62. Sur les qualités du prince idéal, J. KRYNEN, Idéal du prince et pouvoir royal en France à la fin du Moyen Âge, Paris, 1981, p. 73-136.

63. La traduction du Liber de informatione principum éditée en 1517 chez Guillaume Eustache est connue sous un titre qui attribue faussement l'œuvre à Gilles de Rome : Le Mirouer exemplaire et tres fructueuse instruction selon la compillation de Gilles de Rome. Y. DE SAINT-LÉGER, Le miroir des dames, C. MARAZZA éd., Milella-Lecce, 1978.

64. Érudition, fo $21 \mathrm{v}^{\mathrm{o}}$.

65. M.-T. GousSET, F. AVRIL et J. RICHARD, Saint Louis, roi de France. Livre des faits de Monseigneur saint Louis, BnF ms. fr. 2829, Paris, 1990, p. 8-9 et p. 84-88.

66. Espérance : le mécénat religieux des ducs de Bourbon à la fin du Moyen Âge, F. PERROT éd., Souvigny, 2001, p. 12-15, 23 et 57. C. BILLOT, Les saintes chapelles royales et princières, Paris, 1998 , p. 51 et suiv. Fondations de Louis de Bourbon-Montpensier à Aigueperse et de Louis de Bourbon, prince de La Roche-sur-Yon, à Champigny-sur-Veude.

67. Érudition, $\mathrm{f}^{\circ} 3 \mathrm{v}^{\circ}$. Robert le Pieux ( $\mathrm{f}^{\circ} 20$ et $\mathrm{f}^{\circ} 36 \mathrm{v}^{\circ}$ ), Charlemagne ( $\mathrm{f}^{\circ} 38$ ), saint Louis (fo 20 et $\mathrm{f}^{\circ} 38 \mathrm{v}^{\circ}$ ). En outre, le manuscrit conserve une copie des enseignements de saint Louis à son fils (cf. note 24).

68. Le duc Louis II (1337-1410), comte de Clermont et de Forez, épouse Anne, dauphine d'Auvergne.

69. Érudition, $\mathrm{f}^{\circ} 21 \mathrm{v}^{\mathrm{o}}$. Il s'agit de la bataille de Mahdia, un demi-échec qui profite aux Génois. A. LEGUAI, « Le duc de Bourbon Louis II et la croisade de Barbarie, 1390 », Études bourbonnaises, $\mathrm{n}^{\circ} 282,1998$, p. 217-225.

70. Sur Louis II, voir O. MATTÉONI, « Portrait du prince idéal et idéologie nobiliaire dans la "Chronique du bon duc Loys de Bourbon" (1429) ", Studi Francesi, 115, 1995, p. 1-23 et "L'image du duc Louis II de Bourbon dans la littérature du temps de Charles VI », dans Saint-Denis et la royauté. Études offertes à Bernard Guenée, Paris, 1999, p. 145-156. J. CABARET D'ORVILle, La chronique du bon duc Loys de Bourbon, A.-M. CHAZAUd éd., Paris, 1876, en particulier p. 273, le goût du duc pour les livres d'histoire. Ses livres sont encore mentionnés en 1474 à Aigueperse. A. DE BoISLISLE, « Inventaire des bijoux, vêtements, manuscrits et objets précieux appartenant à la comtesse de Montpensier (1474) ", Annuaire-bulletin de la Société de l'Histoire de France, t. XVII, 1880, p. 306 et suivantes ; C. BEAUNE et E. LEQUAIN, « Femmes et histoire en France au Xv ${ }^{\mathrm{e}}$ siècle : Gabrielle de la Tour et ses contemporaines ", Médiévales, 38, printemps 2000, p. 128.

71. Enseignements, p. 49. Sur l'importance donnée aux ancêtres, voir C. KLAPISCH-ZUBER, L'ombre des ancêtres. Essai sur l'imaginaire médiéval de la parenté, Paris, 2000. 
72. J. JACQUART, François I ${ }^{\text {er }}$, Paris, rééd. 1994, p. 23. Nomination au 12 janvier 1515. 73. En 1517 le roi François ${ }^{\mathrm{er}}$ n'a que des filles : Louise (1515) et Charlotte (1516). En novembre 1517, il se rend à Saint-Martin à Tours pour demander un fils qui naît en février 1518. Depuis 1516, le connétable Charles ne reçoit plus de pensions mais le roi devient le parrain de son fils en 1517. En 1521, sa défaveur apparaît lors de la campagne contre Charles Quint et la mort de Suzanne ouvre la délicate succession bourbonnaise revendiquée par Louise de Savoie. R. J. KNECHT, Un prince de la Renaissance. François $I^{\text {er }}$ et son royaume, Paris, 1998, p. 122 et p. 203-204.

74. A.-M. LECOQ, François I er imaginaire. Symbolique et politique à l'aube de la Renaissance française, Paris, 1987, p. 261 et suivantes.

75. A. DE BoISLISLE, « Projet de croisade du premier duc de Bourbon, 1316-1333 », Annuaire-bulletin de la Société de l'Histoire de France, 1872, p. 230-236 et 246-255. A. LEGUAI, « Bourbonnais et Auvergnats à la Croisade de Prusse ", Études Bourbonnaises, n 258 , 1991, p. 93-97.

76. Érudition, fo 41.

77. Sur la date de naissance de François de Bourbon, cf. note 20.

78. G. DE MARILLAC, op. cit., p. 168. J.-M. DE LA MURE, op. cit., p. 551. Jean Le Véneur, évêque et comte de Lisieux depuis 1505, abbé du Bec et du Mont Saint-Michel, lieutenant-général au gouvernement de Normandie en 1525, est grand aumônier du roi en 1526, cardinal en 1533 et meurt en 1543. L. MORERI, Le Grand dictionnaire historique, t. 10, 1759. G. MinoIs, Le confesseur du roi. Les directeurs de conscience sous la monarchie française, Paris, 1988, p. 256.

79. Enseignements, p.103-104. Anne est la marraine de François de Bourbon.

80. Le prénom de François d'Angoulême s'explique par l'intercession de saint François de Paule probablement requise par Louise de Savoie pour avoir un fils. Claude de France en aurait fait autant. A.-M. LECOQ, op. cit., p. 435 et suivantes. Pour l'enfant tant attendu de Suzanne, le prénom convenait à double titre.

81. M. CHOMBART DE LAUWe, Anne de Beaujeu ou la passion du pouvoir, Paris, 1980, p. 432-3. P. PRADEL, op. cit., Paris, 1986, p. 202. J.-M. DE LA MURE, op. cit., p. 551-552. S. CHAMPIER, Les gestes ensemble la vie du preulx Chevalier Bayard, D. CROUZET éd., Paris, 1992, livre III, ch. 3, p. 195-196.

82. Sur le lien de parrainage, A. FINE, Parrains, marraines. La parenté spirituelle en Europe, Paris, 1994. Un autre cas de baptême suivi d'un adoubement dans G. MiNoIs, Du Guesclin, Paris, 1993, p. 387. Le 15 mars 1372, Bertrand Du Guesclin porte sur les fonts Louis de Touraine, futur duc d'Orléans, et l'arme chevalier.

83. Paris, BnF, ms. fr. 2285 , Le compas du dauphin, fo $5 \mathrm{v}^{\circ}$, cité par A.-M. LECOQ, op. cit., p. 77.

84. M. NASSIET, Parenté, noblesse et États dynastiques, $\mathrm{XV}^{e}-\mathrm{XVI}^{e}$ siècles, Paris, 2000, p. 310 et suivantes. La duchesse eut des jumeaux qui ne vécurent pas.

85. Louis XI fait composer le Rosier des guerres pour le futur Charles VIII.

86. La place faite à l'histoire nationale est une nouveauté des miroirs faits pour les Valois. J. KRYNEN, L'empire du roi, op. cit., p. 190-1.

87. D'ailleurs Suzanne autorise avant 1521 l'impression des Enseignements écrits par sa mère. 


\section{RÉSUMÉS}

Au début du XVI ${ }^{\mathrm{e}}$ siècle, Suzanne de Bourbon reçoit les célèbres enseignements écrits par sa mère Anne de France peu de temps avant son mariage (1505), et un traité compilé par le frère prêcheur Pierre Martin à l'annonce de sa première grossesse (1517). Ces textes s'inscrivent dans une longue tradition didactique écrite pour la noblesse et ils coïncident avec une période délicate pour le puissant duché de Bourbon. Ils témoignent de l'importance accordée à l'éducation des enfants de la noblesse, un domaine dans lequel la maison ducale de Bourbon jouit d'une très bonne réputation. Suzanne se doit d'être à la fois une princesse exemplaire et une mère capable de transmettre correctement la vertu de noblesse à sa descendance.

The House of Bourbon, "school of virtue and perfection ». Anne of France, Suzanne of Bourbon and Pierre Martin. In the early 16th century, Suzanne of Bourbon received the famous teachings written by her mother Anne of France a short time before her wedding (1505) and a treatise compiled by the dominican Pierre Martin when the duchess was pregnant for the first time (1517). These texts took place in the long didactic tradition of texts written for the nobility and they coincided with a sensitive period for the powerful duchy of Bourbon. They attested the importance given to the education of the noble children, a field in which the house of Bourbon had a very good reputation. Suzanne had to be both an exemplary princess and a mother able to properly transmit the virtue of nobility to her descendants.

\section{INDEX}

Mots-clés : Anne de France, Suzanne de Bourbon, education, femmes

Keywords : Pierre Martin, Anne of France, Suzanne of Bourbon, women

\section{AUTEUR}

\section{ÉLODIE LEQUAIN}

Université de Paris X-Nanterre, Département d'Histoire, 200, avenue de la République, F-92000 Nanterre 